\title{
Evaluation of the incidence, risk factors, severity, and outcome of hydrocephalic fetuses in a tertiary healthcare center located in Northern India: a prospective observational study
}

\author{
Aditi Jain Garg ${ }^{1 *}$, Rehana Najam ${ }^{1}$, Ritika Agarwal $^{1}$, Rajul Rastogi ${ }^{2}$
}

\begin{abstract}
${ }^{1}$ Department of Obstetrics and Gynecology, Teerthanker Mahaveer Medical College and Research Centre, Moradabad, Uttar Pradesh, India

${ }^{2}$ Department of Radiodiagnosis, Teerthanker Mahaveer Medical College and Research Centre, Moradabad, Uttar Pradesh, India
\end{abstract}

Received: 15 November 2019

Revised: 29 November 2019

Accepted: 09 December 2019

\section{*Correspondence: \\ Dr. Aditi Jain Garg, \\ E-mail: aditijain1205@gmail.com}

Copyright: (c) the author(s), publisher and licensee Medip Academy. This is an open-access article distributed under the terms of the Creative Commons Attribution Non-Commercial License, which permits unrestricted non-commercial use, distribution, and reproduction in any medium, provided the original work is properly cited.

\begin{abstract}
Background: Hydrocephalus is a heterogeneous disease marked by abnormal dilatation of the cerebral ventricles secondary to varying etiologies. This study was aimed to determine the incidence, risk factors, severity, and outcome of hydrocephalic fetuses presenting to a tertiary healthcare hospital located in northern India.

Methods: In this prospective observational study, pregnant women visiting the obstetrics outpatient department of our hospital from 01 July 2017 to 31 June 2018 were screened for hydrocephalic fetuses via ultrasonography along with a detailed history, and a comprehensive battery of diagnostic investigations. They were followed up for a minimum period of two months after delivery/termination of pregnancy.

Results: A total of 3627 pregnant women were screened, of which 10 had hydrocephalic fetuses in the observed time period. The incidence of hydrocephalus was determined to be 2.75 per 1000 live births. Low socio-economic status was identified as a major risk factor. 50\% of the hydrocephalic fetuses were severely afflicted and were discontinued. The remaining 50\% were successfully delivered and were managed via a ventriculo-peritoneal shunt or are under close observation in the postnatal period without any adverse outcome.

Conclusions: The burden of hydrocephalus is considerably high in India, as compared to western countries. In rural settings, low socioeconomic status and lack of folic acid supplementation have a major influence in the etiopathogenesis of hydrocephalus. Management of hydrocephalus requires a multidisciplinary approach and is tailored according to the severity of the presentation. Severe cases of hydrocephalus and cases with associated anomalies have a poor prognosis.
\end{abstract}

Keywords: Low socioeconomic status, Prenatal hydrocephalus, Ventriculomegaly, Ventriculo-peritoneal shunt

\section{INTRODUCTION}

Hydrocephalus is a heterogeneous disease marked by abnormal dilatation of the cerebral ventricles secondary to varying etiologies. ${ }^{1}$ It is the most common cranial abnormality encountered at prenatal ultrasonography, with a worldwide incidence ranging from 0.68 to 3.16 per thousand live births. ${ }^{1-3}$ The burden of prenatal hydrocephalus is high in developing countries as India. However, its incidence in rural settings of India is 
relatively unknown. The etiology of hydrocephalus is multifactorial which includes presence of intracranial malformations (neural tube defects, aqueductal stenosis, etc), genetic mutations, hemorrhage, infections, and tumors. ${ }^{3-5}$ Several risk factors such as low birth weight, prematurity, gestational diabetes mellitus, and ethnicity have been implicated in its etiopathogenesis. ${ }^{6,7}$

Ultrasonography is the gold standard for diagnosing congenital hydrocephalus. ${ }^{8}$ Depending on the extent of ventriculomegaly and presence of other congenital anomalies, hydrocephalus is classified as mild, moderate, or severe. ${ }^{8,9}$ Literature about the clinical outcome of congenital hydrocephalus according to its severity is sparse. ${ }^{9,10}$

Considering all the above-mentioned facts, the present study was aimed to determine the incidence, risk factors, severity, and outcome of congenital hydrocephalus in our institute, which is a tertiary healthcare center located in northern India.

\section{METHODS}

The present study is prospective observational in nature. It was conducted in the department of obstetrics and gynecology, at our tertiary care centre from $1^{\text {st }}$ July 2017 to $31^{\text {st }}$ June 2018.

\section{Inclusion criteria}

- All pregnant women, who had attended our antenatal outpatient department and/or had delivered in our institute in the specified time duration.

\section{Exclusion criteria}

- Women who refused to give consent for participation or were lost to follow up.

The included women were screened for hydrocephalic fetuses via detailed ultrasonography.

For annual incidence rate determination, the denominator comprised of all women, who met the screening criteria. Annual incidence rate of prenatal hydrocephalus per 1000 live births was calculated using the formula mentioned below.

$$
\begin{aligned}
& \text { Incidence } \\
& =\frac{\text { Number of confirmed cases of hydrocephalus in the year }}{\text { Number of pregnant women screened in the year }}
\end{aligned}
$$

A case of fetal hydrocephalus was defined as anterior horn width $>10 \mathrm{~mm}$ across the atria of the lateral ventricles, as measured by ultrasonography on an axial section of the head. Hydrocephalus was further defined as mild $(10-12 \mathrm{~mm})$, moderate $(12-15 \mathrm{~mm})$, or severe $(>15$ $\mathrm{mm})$ according to the extent of ventriculomegaly. ${ }^{10-12}$ Ultrasonography was also used to diagnose associated congenital anomalies. Confirmed cases with a minimum follow-up period of two months of were included in the final analyses. An informed written consent was mandatory.

Demographic details, and information about the observed risk factors were obtained via detailed history and examination. The risk factors observed in our study were decided after reviewing the literature. ${ }^{3-7}$ Low socioeconomic status was defined as per the Kuppuswamy scale. ${ }^{13}$

Management of both mother and child was tailored according to the gestational age and associated medical conditions. Confirmed hydrocephalic fetuses and their mothers were followed up for a minimum period of two months after delivery/termination of pregnancy to determine their respective outcomes. Outcome of both mother and hydrocephalic child was classified according to the severity of ventriculomegaly.

All procedures performed in our study were in accordance with ethical standards of the institutional ethics committee of Teerthanker Mahaveer Medical College and Research Centre and with the 1964 Helsinki declaration and its later amendments. Informed consent was obtained from all individual participants included in the study.

\section{Statistical analysis}

Due to the small size of our sample, we conducted a descriptive statistical analysis. As all our variables were qualitative, we expressed the data as percentages with $95 \%$ confidence intervals.

\section{RESULTS}
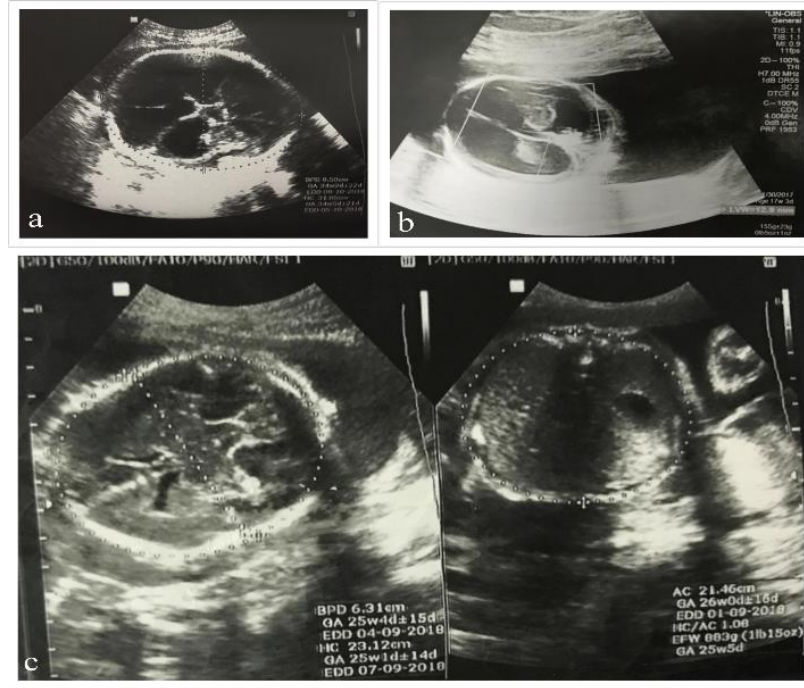

Figure 1: Ultrasonography findings of fetuses with mild (a), moderate (b), and severe

(c) ventriculomegaly. 
A total of 3627 pregnant women were screened for hydrocephalic fetuses in the specified time period, of which 10 confirmed cases were identified. There were 2 cases of mild, 4 cases of moderate, and 4 cases of severe ventriculomegaly, respectively (Figure 1). The annual incidence was calculated to be 2.75 per 1000 live births.

Table 1: Identified etiologies of the hydrocephalic fetuses (based on ultrasound imaging).

\begin{tabular}{|lccc|}
\hline Etiology & Mild (2) & Moderate (4) & Severe (4) \\
\hline Congenital malformations & & & - \\
\hline Myelomeningocele (Arnold Chiari malformation type-II) & 1 & & \\
\hline Syndromes & - & - & 1 \\
\hline Dandy Walker syndrome & 1 & 1 & - \\
\hline Down syndrome & & - & 2 \\
\hline Miscellaneous & - & 2 & 1 \\
\hline Intraventricular hemorrhage & - & & \\
\hline Idiopathic & & & \\
\hline Values mentioned represent number of fetuses.
\end{tabular}

Table 2: Risk factors for hydrocephalus (according to the degree of ventriculomegaly).

\begin{tabular}{|c|c|c|c|c|}
\hline Risk factors & Mild (2) & Moderate (4) & Severe (4) & Total (10) \\
\hline Age $\geq 30$ years & 0 & 2 & 2 & 4 \\
\hline Low SE status & 2 & 3 & 4 & 9 \\
\hline No folic acid supplementation & 1 & 3 & 4 & 8 \\
\hline GDM & 1 & 1 & 0 & 2 \\
\hline Alcohol history & 0 & 1 & 0 & 1 \\
\hline Drug history & 0 & 0 & 1 & 1 \\
\hline Presence of other anomalies & & & & 5 \\
\hline Arnold Chiari malformation & 1 & 1 & 0 & - \\
\hline Dandy Walker malformation & 0 & 0 & 1 & - \\
\hline Down syndrome & 1 & 1 & 0 & - \\
\hline \multicolumn{5}{|l|}{ Period of gestation at diagnosis } \\
\hline 14-24 weeks & 1 & 2 & 1 & 4 \\
\hline 25-34 weeks & 1 & 2 & 1 & 4 \\
\hline$\geq 34$ weeks & 0 & 0 & 2 & 2 \\
\hline
\end{tabular}

Ultrasound findings suggestive of down syndrome in two of the hydrocephalic fetuses were increased nuchal fold thickness, short femoral length, and echogenic bowel. Other soft markers of aneuploidy could not be detected, as most of the fetuses were diagnosed after 25 weeks of gestation. Ultrasonography of one of the hydrocephalic fetuses showed absence of cerebellar vermis, cystic dilation of the fourth ventricle, and marked enlargement of the cisterna magna. Hence, it was diagnosed as a fetus with Dandy Walker syndrome. Sonographic findings of two fetuses depicted a small posterior cranial fossa, presence of the lemon and banana cerebellum signs, along with the presence of a meningomyelocele. Hence, they were both classified as fetuses with Arnold Chiari malformation type-II. The mutations leading to Dandy Walker malformation, and Arnold Chiari malformation could not be identified. Ultrasound images of two hydrocephalic fetuses in the third trimester were consistent with intraventricular hemorrhage, the causes of which could not be pinpointed. Also, the causes of ventriculomegaly in three of the fetuses could not be identified through ultrasound imaging (Table 1).

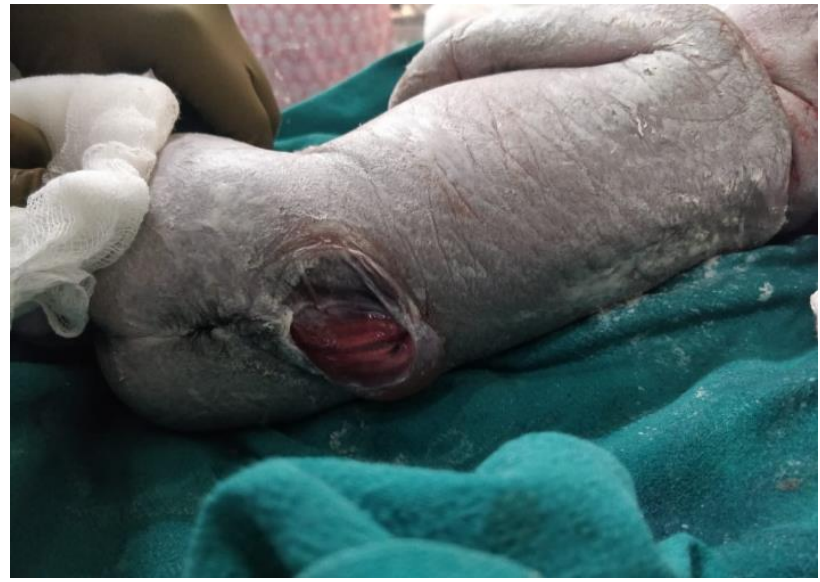

Figure 2: A terminated fetus with severe ventriculomegaly and associated neural tube defects. 
The risk factors which were found to be maximally associated with hydrocephalus in our study were low socioeconomic status (90\%), lack of folic acid supplementation $(80 \%)$, presence of other congenital anomalies (50\%), age > 30 years $(40 \%)$, and diagnosis before 24 weeks of gestation (40\%). Other risk factors are mentioned in Table 2 .

A total $50 \%(\mathrm{n}=5)$ of the fetuses were terminated, and $50 \%(\mathrm{n}=5)$ survived (Figure 2 and Figure 3$)$.

The termination rate was high in the severely affected group $(\mathrm{n}=3)$, as compared to the other groups. The surviving fetuses were delivered via vaginal delivery $(\mathrm{n}=$ $2)$ or cesarean section $(n=3)$. After 2 months of followup, it was observed that the surviving fetuses underwent a ventriculo-peritoneal shunt $(\mathrm{n}=3)$ or are under close observation in the postnatal period without any adverse outcome $(n=2)$. All the mothers had a favourable outcome and survived $(\mathrm{n}=10)($ Table 3$)$.

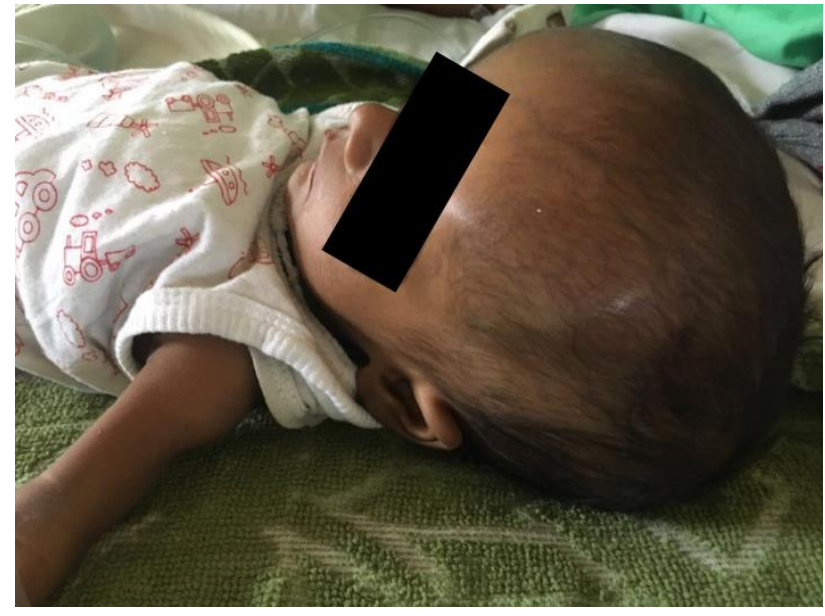

Figure 3: A continued fetus with moderate ventriculomegaly managed by a ventriculo-peritoneal shunt in the postnatal period.

Table 3: Outcomes of child and mother after 2 months of follow-up.

\begin{tabular}{|c|c|c|c|c|c|c|}
\hline \multirow{2}{*}{$\begin{array}{l}\text { Severity of } \\
\text { hydrocephalus }\end{array}$} & \multicolumn{4}{|c|}{ Outcome of child } & \multicolumn{2}{|c|}{ Outcome of mother } \\
\hline & MTP & Survived & Vaginal delivery & Caesarean section & Mortality & Survived \\
\hline Mild $(n=2)$ & 1 & 1 & $1^{*}$ & 0 & 0 & 2 \\
\hline Moderate $(n=4)$ & 1 & 3 & $1^{*}$ & $2^{*}$ & 0 & 4 \\
\hline Severe $(n=4)$ & 3 & 1 & 0 & $1^{*}$ & 0 & 4 \\
\hline
\end{tabular}

MTP: medical termination of pregnancy; * Underwent a ventriculo-peritoneal shunt $(n=3)$; + Under observation in the postnatal period without an adverse outcome $(n=2)$.

\section{DISCUSSION}

Hydrocephalus is a complex condition caused by physical or functional obstruction of cerebrospinal flow that leads to progressive ventriculomegaly., ${ }^{1,3}$ It is traditionally classified as obstructive or communicating. ${ }^{2,3}$ The worldwide incidence of hydrocephalus varies between 0.68 to 3.16 per 1000 live births. ${ }^{2}$ In our study we estimated the incidence to be 2.75 per 1000 live births, which was comparatively on the higher side. This finding is consistent with previous reports which suggest that the burden of hydrocephalus is highest in low- and middleincome countries including India and is often underreported..$^{1,2}$

The etiopathogenesis of hydrocephalus is both convoluted and multifactorial. Extrinsic causes of hydrocephalus include hemorrhage, neoplasm, infection (bacterial meningitis, CMV infection, toxoplasmosis.), and drug/alcohol intake. ${ }^{3}$ Developmental hydrocephalus is often associated with several pediatric syndromes comprising of anomalies such as myelomeningocele, aqueductal stenosis, posterior fossa crowding, etc. ${ }^{8}$ Numerous genes such as L1CAM, AP1S2, POMT, MPDZ, FUZ, VANGL1, CELSR1, OFD1, GPSM2, FGFR, and NF1 have also been implicated in its pathogenesis. $^{3,5}$
Once the diagnosis is confirmed via ultrasonography, the parent's apprehension needs to be tackled with great care. They need to be counselled in details about the risks of continuing the pregnancy based on the severity of the hydrocephalus, and motivated for further investigations to improve the fetal outcome. ${ }^{14,15}$

Investigations such as detailed ultrasonography, karyotyping, chromosomal analysis, TORCH screening, and fetal magnetic resonance imaging are often employed to determine the underlying etiology, and identify other fetal anomalies. ${ }^{8,10,16,17}$ In our study, we identified intraventricular hemorrhage, Dandy Walker syndrome, Arnold Chiari malformation type-II, and Down syndrome as underlying etiologies in some of the cases, based on ultrasound findings. However, most parents couldn't proceed with further investigations to determine the chromosomal/causative basis of these etiologies due to financial constraints.

In western countries, the major risk factors identified to be associated with congenital hydrocephalus include lack of prenatal care, multiparous gestation, maternal diabetes, maternal chronic hypertension, maternal hypertension during gestation, low socioeconomic status, and alcohol/drug use during pregnancy. ${ }^{6}$ However, in our study we found low socioeconomic status and lack of 
folic acid supplementation as leading risk factors associated with hydrocephalus. This finding could be explained by the fact that most of the pregnant women referred to our center are of low socioeconomic status. Low socioeconomic status directly/indirectly influences prenatal care which includes folic acid supplementation. ${ }^{1,7}$ Most pregnant women visiting our hospital did not have any prior antenatal visit and $60 \%$ of fetuses were diagnosed with hydrocephalus after 24 weeks of gestation, indicating lack of prenatal care. Other risk factors which we found relevant and consistent with previous studies included maternal age, maternal diabetes, maternal drug/alcohol history, and diagnosis before 34 weeks of gestation. ${ }^{7,8,10}$ However, further research is warranted to confirm the impact of these identified risk factors and examine their behavioral and/or biological basis to develop suitable preventive measures.

Isolated mild to moderate ventriculomegaly is linked to an abnormal outcome in $10-20 \%$ of children. ${ }^{8,10}$ In our study, all cases of isolated mild to moderate ventriculomegaly $(n=2)$ were successfully managed with a ventriculo-peritoneal shunt in the postnatal period. Generally, fetuses with isolated severe vetriculomegaly are considered to have a poor prognosis. ${ }^{8,12,14}$ In our study, we observed that $100 \%$ of parents with such fetuses $(n=3)$ chose to terminate their pregnancies. The high termination rate could be due to the lack of sufficient data regarding the outcome of such fetuses. However, some conflicting reports have suggested that fetuses with isolated severe ventriculomegaly have a favorable outcome in almost $60 \%$ of the cases. ${ }^{9}$

Ventriculomegaly with associated anomalies is characterized by an abnormal outcome in up to $40-50 \%$ of children. ${ }^{8,12,17}$ In our study, $60 \%(\mathrm{n}=3)$ of all hydrocephalic fetuses associated with other congenital anomalies $(n=5)$ were terminated. The remaining $40 \%$ $(n=2)$ were delivered successfully without any major abnormal outcome in the follow-up period.

Timing and mode of delivery in each of the pregnancies was selected on the basis of standard obstetrics indications taking into account both maternal and fetal complications. ${ }^{3,6,7}$ In our study, mode of delivery was surgical in $60 \%$ of the cases, and vaginal in $40 \%$ of the cases. In one patient vaginal birth after cesarean section (VBAC) was preferred as the presentation of the baby was vertex with mild macrocephaly. Cephalocentesis may be an option to potentially allow vaginal delivery. ${ }^{8}$ In the postpartum follow-up period, all mothers recovered without complications indicating a positive maternal outcome.

Management of hydrocephalus requires a multidisciplinary approach and is tailored according to the etiology, and severity of the disease. ${ }^{3,7,10}$ Current long-term treatment options for hydrocephalic children are restricted to surgical interventions, including shunt placement and endoscopic ventriculostomy. ${ }^{10,16,18}$ Noninvasive treatment options including use of diuretics/fibrinolytics and lumbar puncture only provides temporary relief. ${ }^{19}$

Fetal procedures conducted in the intrauterine life in order to improve outcome include cephalocentesis, vetriculo-amniotic shunt, and fetal neuroendoscopic procedures. ${ }^{8}$ However, literature about the success rate of these procedures is sparse.

Our study had numerous limitations. First, the duration of the study was short due to which we had a few numbers of cases. Second, due to the financial constraints of the patients we could not conduct detailed radiological/molecular investigations to determine the genetic/causative basis of the underlying etiologies. Third, due to the short follow-up period we could not determine the long-term outcome of the cases. Future long-term studies are warranted to confirm our findings.

\section{CONCLUSION}

The incidence of hydrocephalus is considerably high in India. In rural settings of India, low socioeconomic status and lack of folic acid supplementation are leading risk factors associated with hydrocephalus. Outcome of patients with isolated mild to moderate hydrocephalus is generally favourable. Severe cases of hydrocephalus and cases with associated anomalies have a poor prognosis.

\section{Funding: No funding sources}

Conflict of interest: None declared

Ethical approval: The study was approved by the Institutional Ethics Committee

\section{REFERENCES}

1. Isaacs AM, Riva-Cambrin J, Yavin D, Hockley A, Pringsheim TM, Jette N, et al. Age-specific global epidemiology of hydrocephalus: systematic review, metanalysis and global birth surveillance. PLoS One. 2018;13:e0204926.

2. Dewan MC, Rattani A, Mekary R, Glancz LJ, Yunusa I, Baticulon RE, et al Global hydrocephalus epidemiology and incidence: a systematic review and meta-analysis. J Neurosurg. 2018;27:1-15.

3. Tully HM, Dobyns WB. Infantile hydrocephalus: a review of epidemiology, classification and causes. Eur J Med Genet. 2014;57:359-68.

4. Tully HM, Ishak GE, Rue TC, Dempsey JC, Browd SR, Millen KJ, et al. Two hundred thirty-six children with developmental hydrocephalus: causes and clinical consequences. J Child Neurol. 2016;31(3):309-20.

5. Zhang J, Williams MA, Rigamonti D. Genetics of human hydrocephalus. J Neurol. 2006;253:1255-66.

6. Kalyvas AV, Kalamatianos T, Pantazi M, Lianos GD, Stranjalis G, Alexiou GA. Maternal environmental risk factors of congenital 
hydrocephalus: a systematic review. Neurosurg Focus. 2016;41:E3.

7. Venkataramana NK. Hydrocephalus Indian scenario: a review. J Pediatr Neurosci. 2011;6:S11-S22.

8. Cavalheiro S, Silva da Costa MD, Mendonca JN, Dastoli PA, Suriano IC, Barbosa MM, et al. Antenatal management of neurosurgical diseases. Childs Nerv Syst. 2017;33:1125-41.

9. Letouzey M, Chadie A, Brasseur-Daudruy M, Proust F, Verpyck E, Boileau P, et al. Severely apparently isolated fetal ventriculomegaly and neurodevelopmental outcome. Prenat Diagn. 2017;37:820-6.

10. Fox NS, Monteagudo A, Kuller JA, Craigo S, Norton ME, Society for Maternal-Fetal Medicine (SMFM. Mild fetal ventriculomegaly: diagnosis, evaluation, and management. Am J Obstet Gynecol 2018;219:B2-9.

11. Bijarnia-Mahay S, Puri RD, Kotecha U, Dash P, Pal $\mathrm{S}$, Lall M, et al. Outcome of prenatally-detected fetal ventriculomegaly. J Fetal Med. 2015;2(1):39-44.

12. Gagilioti P, Danelon D, Bontempo S, Mombro M, Cardaropoli S, Todros T. Fetal cerebral ventriculomegaly: outcome of 176 cases. Ultrasound Obstet Gynecol. 2015;25:372-7.

13. Sharma R, Saini NK. A critical appraisal of Kuppuswamy's socioeconomic status scale in the present scenario. J Family Med Prim Care. 2014;3:34.
14. Breeze AC, Alexander PM, Murdoch EM, Missfelder-Lobos HH, Hackett GA, Lees CC. Obstetric and neonatal outcomes in severe fetal ventriculomegaly. Prenat Diagn. 2007;27:124-9.

15. Chiu TH, Haliza G, Lin YH, Hung TH, Hsu JJ, Lo LM. A retrospective study on the course and outcome of fetal ventriculomegaly. Taiwan J Obstet Gynecol. 2014;53:170-7.

16. Williams MA, Malm J. Diagnosis and treatment of idiopathic normal pressure hydrocephalus. Continuum (Minneap Minn). 2016;22:579-99.

17. Twining $\mathrm{P}$, Jaspan T, Zuccollo J. The outcome of fetal vetriculomegaly. Br J Radiol. 1994;67:26-31.

18. Smith J, Cheater F, Bekker H. Parent's experiences of living with a child with hydrocephalus: a crosssectional interview-based study. Health Expect. 2015;18:1709-20.

19. Del Bigio MR, Di Curzio DL. Nonsurgical therapy for hydrocephalus: a comprehensive and critical review. Fluids Barriers CNS. 2016;13:3.

Cite this article as: Garg AJ, Najam R, Agarwal R, Rastogi R. Evaluation of the incidence, risk factors, severity, and outcome of hydrocephalic fetuses in a tertiary healthcare center located in Northern India: a prospective observational study. Int J Reprod Contracept Obstet Gynecol 2020;9:595-600. 